\title{
Toll-Like Receptors-The Guardians at the Interface
}

\author{
Syed Mohmad Shah ${ }^{1}$ and Arem Qayum ${ }^{2}$ \\ ${ }^{1}$ Sher-e-Kashmir University of Agricultural Sciences and Technology of Kashmir, J\&K, India \\ ${ }^{2}$ CSIR-IIIM Jammu Canal Road Jammu, J\&K, India \\ *Corresponding author
}

\section{A B S T R A C T}

\section{Keywords}

Toll-like receptors, Innate immunity, Cytokines,

Adaptive immunity, TLR signaling

Article Info

\section{Accepted:}

20 June 2021

Available Online:

10 July 2021
TLRs, named for their homology to Drosophila protein Toll, are a class of single membrane- spanning non-catalytic receptors that recognize structurally conserved molecules derived from microbes, once they have breached the physical barriers such as skin and intestinal mucosa, and activate immune cell responses. They play an important role in innate immunity by recognizing a spectrum of PAMPs, by directly and indirectly inducing innate and adaptive immune responses and by having their spatial expression coincident with the host's environmental interface. The main players in innate immunity, phagocytes, neutrophils, macrophages and dendritic cells, discriminate between pathogens and the self by utilizing signals from the TLRs. They participate in the translation of the non specific information contained in conserved PAMPs into antigen-specific information and clonal expansion of $\mathrm{T}$ cells, there by tailoring the adaptive immune response. Mammalian TLRs comprise a large family of at least 11 members. Since, TLRs bind to the ligands that are indispensable components of pathogens, they hold a promise to provide effective vaccine strategies to deal with the mutating ability of pathogens by which they overcome the antibiotic sensitivity and vaccination failures.

\section{Introduction}

Activation of innate immunity relies on nonclonal and germ- line- encoded pattern recognition receptors (PRRs) that recognize conserved microbial molecular patterns known as microbial /pathogen associated molecular patterns (PAMPs/MAMPs). PRRs have evolved to take advantage of three salient PAMP qualities viz; constitutive expression, class specificity and mutation resistance, there by forming the first-line host defense against the invading pathogens(Janeway and Medzhitov, 2002). They recognize pathogens via their PAMPs, recruit other extracellular and intracellular proteins to the complex, initiate signaling cascades that ultimately impact transcription, activate complement and are further involved in coagulation, phagocytosis, inflammation and apoptosis 
functions in response to pathogen detection. There are several types of PRRs like complement, glucan-, mannose-, scavengerand Toll - like receptors, each with specific PAMP ligand, expression pattern, signaling pathways and anti- pathogen responses (Medzhitov, 2001).

\section{Toll-like receptors}

The protein Toll first attracted attention during 1980s, when researchers in Germany found that developing flies could not establish a proper dorso-ventral axis without Toll. Toll refers to the mutant flies' bizarrely scrambled anatomy. It means "weird" in German slang. In 1996, Jules Hoffmann and Bruno Lemaitre found that mutations in Toll made flies highly susceptible to lethal infection with Aspergillus fumigates. The Drosophila Toll family counts nine members, Toll, 18-Wheeler, and Toll-3 to 9 (Ooi et al., 2002). The related molecules in vertebrates with roles in innate immunity came to be known as Toll- like receptors (TLRs). The Toll like receptor multi-gene family encodes important recognition receptors that form the primary component of the afferent arm of the innate immunity (Pichlmair and Reis, 2007). Data base searches have identified a family of human Toll homologues whose intracellular domain resembles that of mammalian $\mathrm{IL}-1$ receptor (IL-1R), there by defining the Toll/IL-1R receptor family (Schwandner et al., 1999). IL1R/ TLR super family is composed of three subgroups. Members of the first group, IL-1, IL-8 and ST2, possess immunoglobulin domains in their extracellular region. The second group encompasses the TLRs, whose ectodomain, is composed of leucine-rich repeats (LRRs), flanked by characteristic cysteine- motifs. The third group includes intracellular adaptor proteins important for signaling. TLRs are a class of type 1 single membrane-spanning non-catalytic receptors that recognize structurally conserved molecules derived from microbes, once they have breached the physical barriers such as skin and intestinal mucosa. Phagocytes, neutrophils, macrophages and dendritic cells, discriminate between pathogens and the self by utilizing signals from TLRs. TLRs and members of the IL-1R family share a conserved stretch of approximately 200 aminoacids in their cytoplasmic region known as Toll/IL-1R (TIR) domain. The region of homology in TIR motif is confined to three conserved boxes that contain amino acids crucial for signaling. The extracellular portion of TLRs contains a leucine-rich repeat (LRR) motif, whereas that of the IL-1Rs contains three immunoglobulin domains. The LRR domains consist of varying numbers of repeats, each 24-29 aminoacids in length, containing the motif $X X \mathrm{~L} X \mathrm{~L} X X$ ( $\mathrm{X}$ is any amino acid and $\mathrm{L}$ is leucine) and other conserved leucine residues. These LRR domains are directly involved in the recognition of a variety of pathogens (Akira, 2003). Mammalian TLRs comprise a large family of at least 11 members. TLR1- 9 are conserved between human and mouse. TLR10 is functional in human but not in mouse. The C-terminal half of the mouse TLR10 gene is substituted to an unrelated and nonproductive sequence. The ligands that bind TLRs are indispensable components of pathogens, e.g., nucleic acid of viruses, LPS of Gram-negative bacteria, peptidoglycan of Gram-positive bacteria and Zymosan of fungi. Pathogens simply do not have the option of mutating the forms that lack the building blocks recognized by TLRs. Hence TLRs are a promise to provide effective strategies to deal with the mutating ability of pathogens by which they overcome the antibiotic sensitivity and vaccination failures.

\section{Cellular compartmentalization of TLRs}

TLRs that recognize extracellular ligands are located on the surface of cells. These include 
TLR-1, 2, 4, 5 and 6. Those that recognize intracellular ligands such as viral RNA or fragments of DNA from bacteria are located in intracellular compartments of cell. These include TLR-3, 7, 8and 9.

\section{Individual Toll-like receptorsTLR1}

TLR1 was cloned out of human erythroleukemic (TF-1) cell line-derived cDNA library (Genbank Acession No. U88540). In humans it maps to chromosome 4 p14 and to BTA 6 (BosTaurus chromosome $6)$ in bovines. It is most closely related to TLR6 and TLR10 with $68 \%$ and $48 \%$ overall amino acid sequence identity, respectively. TLR1 mRNA is ubiquitously expressed and found at higher levels. The functional association of TLR1 with TLR2 to form a heterodimeris involved in recognition of soluble bacterial factors (Mycobacteria, Neisseria, Borrelia) and tri-acylpeptides (Hopkins et al., 2005).

\section{TLR2}

TLR2, also called as Toll/IL-1-like 4 (TIL4) was first cloned using a human lung-derived cDNA library (GenbankAcessionNo.U88878). In humans it maps to chromosome 4q31-32 and to BTA 17 in bovines. It is most closely related to TLR6 with $31 \%$ overall amino acid sequence identity (Chaudhary, 1998). TLR2 forms heterodimers with TLR1, TLR6 and possibly TLR10. TLR2 complexes recognize a wide variety of PAMPs which include lipoproteins/lipopeptides peptidoglycan and lipoteichoicacid, lipoarabinomannan, glycosyl phosphatidylinositolanchors, zymosan and glycolipids from Treponema maltophilum and a number of endogenous ligands viz, necrotic cells and their protein by products and heat shock proteins (HSP60, HSP70 and GP96) (Shah et al., 2012). In addition, TLR2 recognizes LPS preparations from nonenterobacteria such as Leptospira interrogans,
Porphyromonas gingivalis and Helicobacter pyroli (Smith et al., 2003). These LPS structurally differ from the typical LPS of Gram-negative bacteria recognized by TLR4 in the number of acyl chains in the lipid A component, which presumably confers differential recognition (Netea et al., 2002). TLR1 and TLR6 functionally associate with TLR2 and discriminate between diacyl or triacyl lipopeptides. Bacterial lipoprotein (BLP) activation of TLR2 also mediates apoptosis by involving Fas-associated death domain protein (FADD) and caspase 8, indicating that TLR2 is a novel "death receptor" capable of engaging a poptotic machinery without the conventional cytoplasmic death domain (Aliprantis et al., 2000). It was reported by Shah et al., (2012) in one of their studies that TLR2 activation in response to sPGN activation generates a cytokine response, initially of $\mathrm{Th} 2$ type which skews to Th1 type with time, till the stimulated PBMCs become refractory to further stimulation. Identification and sequence analysis of bubaline TLR2 showed that the nucleotide sequences coding for the TLR2 gene were not under positive selection and shared about $98 \%$ identity with that of Capra hircus, Bos indicus and Bison bison (Shah et al., 2010 and Shah et al., 2014).

\section{TLR3}

TLR3 was cloned from a human placentaderived cDNA library. It maps to chromosome 4q35and in bovines to BTA 27. TLR3 is most closely related to TLR5, TLR7 and TLR8, each with $26 \%$ overall aminoacid sequence identity. TLR3 forms a homodimer and recognizes viral double stranded RNA (dsRNA) (Barton and Medzhitov, 2002).

\section{TLR4}

TLR4 was initially cloned as the human homolog of Drosophila Toll (dToll) and thus 
was first named hToll. It maps to chromosome 9q32-33 in humans and to BTA 8 in bovine. It shows high degree of similarity to dToll over the entire aminoacid sequence. TLR4 expression is upregulated on exposure to Gram negative bacteria. TLR4 forms a homodimer and requires the extracellular association of an additional component, MD2. TLR4 complex recognizes lipopolysaccharides, lipoteichoicacid (LTA), certain viruses like respiratory syncytial virus (RSV), hepatitis $\mathrm{C}$ virus (HCV) and mouse mammary tumor virus (MMTV) and endogenous ligands viz., HSPs, fibrinogen, extra domain A of fibronectins, surfactant protein A (SP-A), heparin sulfate, oligosaccharides of hyaluronic acid and $\beta$ defensins. TLR4 is implicated in the recognition of taxol, a diterpene purified from the bark of the western yew (Taxus brevifolia) (Gao and Tsan, 2003).

\section{TLR5}

Full length TLR5 was cloned from PBL- and prostate-derived cDNA libraries and called TIL3, mapping it to chromosome1q41-42 in humans and to BTA16 in bovines.

It is most closely related to TLR3 with $26 \%$ overall amino acid sequence identity. It forms a homodimer as well as a heterodimer with TLR4. Both complexes function to recognize the flagellin protein.

\section{TLR6}

TLR6 was cloned using a human placentaderived cDNA library and the gene mapped to chromosome 4p14 and to BTA 6 in bovines.

It is most closely related to TLR1, TLR10 and TLR2 with $68 \%, 46 \%$ and $31 \%$ overall amino acid sequence identity, respectively. TLR6/2 heterodimer specifically recognizes diacyllipopeptides (Takeda and Akira, 2005).

\section{TLR7}

It was first cloned from a human cDNA library and mapped to human chromosome Xp22 and to BTA X (Bos taurus chromosome $\mathrm{X})$ in bovines. TLR7 is most closely related to TLR 8 and TLR9 with $43 \%$ and $36 \%$ overall amino acid sequence identity, respectively. Its natural ligands are still unknown but it does bind a group of small synthetic antiviral compounds called imidazoquinolines. Murine TLR7 also recognizes another synthetic compound, loxoribine, which has anti- viral and anti- tumor activities. It has been shown that TLR7 and TLR8 recognize guanosineoruridine-rich single stranded RNA (ssRNA) from viruses (Lund et al., 2004).

\section{TLR8}

It was cloned from human placenta cDNA library and mapped to chromosome Xp22 in humans and to BTA $X$ in bovines. It is most closely related to TLR7 and TLR9 with $43 \%$ and $35 \%$ overall aminoacid sequence identity, respectively.

\section{TLR9}

It was cloned from a human placenta cDNA library and mapped to chromosome 3 p21 and to BTA 22 in bovines. It is most closely related to TLR7 and TLR8 with $36 \%$ and $35 \%$ overall aminoacid sequence identity, respectively. TLR9 is essential in recognition of both A/D type and $\mathrm{B} / \mathrm{K}$ type $\mathrm{CpG} \mathrm{DNA}$, viral-derived CpG DNA and pathogenesis of certain autoimmune disorders (Krug et al., 2004).

\section{TLR10}

TLR10 was cloned from a human spleenderived cDNA library. It is most closely related toTLR1 and TLR6 with $48 \%$ and $46 \%$ overall amino acid identity, respectively. TLR10 is present on BTA6 in bovines. 


\section{TLR11}

TLR11 is the most recently identified TLR. It is expressed in bladder epithelial cells and mediates resistance to infection by uropathogenic bacteria in mouse. There is no functionalTLR11protein in humans (Zhang et al., 2004).

\section{TLR expression profile in farm animals}

TLR expression profiles are suggestive of an individual's ability to respond to a challenge. Vahanan et al., (2008) conducted a study to determine the expression profile of TLRs 1-10 in buffalo peripheral blood mononuclear cells (PBMCs), neutrophills, spleen, lung, liver, heart, kidney, ovary and uterus, using reverse transcriptase polymerase chain reaction (RTPCR).

Among the tissues selected for study, TLR1 was found to be expressed in only a few tissues such as neutrophils, spleen, heart, lung and liver. TLR2 was found to be expressed in all cells and tissues analyzed. TLR2 expression was also high in bovine skin, however slightly lower levels were expressed in ovine gut associated tissues (Menzies and Ingham, 2006).

TLR3 was found to be expressed in brain, trachea, PBMCs, spleen, lung, liver, heart and ovary. TLR4 expression was found in spleen, lung, liver, heart, ovary and PBMCs and neutrophils, among the tissues analysed. TLR5 was expressed in all cells and tissues analysed. TLR6 had similar expression profiles as TLR2 except in kidney where TLR2 was expressed and TLR6 was not. TLR7,8 and9 were expressed in all cells and tissues analyzed. TLR10 was expressed in all selected cells and tissues except in heart and kidney. The study revealed that PBMCs express all TLRs exceptTLR1, neutrophils express all TLRs except TLR3, heart lacked an expression of
TLR10 only, kidney had the least repertoire of TLR expression, while ovary expressed all TLRs except TLR1.

\section{The Toll-like receptor family signaling pathway}

Triggering of the TLR causes the adaptor protein MyD88 to be recruited to the receptor complex, which in turn promotes association with the IL-1R-associated kinases, IRAK-4 and IRAK-1. During the formation of this complex, IRAK4 is activated, leading to the hyperphosphorylation of IRAK-1, which then induces the interaction of TRAF6 with the complex. The association of IRAK-4. IRAK1.TRAF6 causes some conformational change in one or more of these factors, leading to their disengagement from the receptor complex. The IRAK-4.IRAK-1.TRAF6 complex then interacts at the membrane with another preformed complex consisting of TAK1, $\mathrm{TAB} 1$, and $\mathrm{TAB} 2$. This interaction induces phosphorylation of TAB2 and TAK1, which then translocate together with TRAF6 and TAB1 to the cytosol. TAK1 is subsequently activated in the cytoplasm, leading to the activation of IKK. This causes phosphorylation and degradation of $\mathrm{I} \kappa \mathrm{B}$ and consequent release of $\mathrm{NF}-\kappa \mathrm{B}$, a ubiquitous transcription factor that regulates the transcription of various genes during inflammatory and immune responses. Activation of TAK1also results in the activation of MAP kinases and c-Jun NH2terminal kinase (JNK). Although MyD88 plays a critical role in TLR signaling, there is a difference in the signaling pathways triggered by LPS and by other bacterial components. Activation of NF- $\mathrm{KB}$ and MAP kinases by mycoplasmal lipopeptide is completely abolished in TLR2- or MyD88deficient macrophages. However, LPS activation of MAP kinases and NF- $\kappa B$ remains intact in MyD88-deficient macrophages, although it is delayed compared with that in 
wild-type mice. This indicates that theTLR4mediated response to LPS may involve both MyD88-dependent and-independent pathways, each of which leads to the activation of MAP

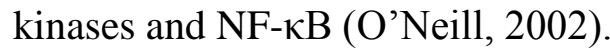

\section{TLRs- Bridge between innate and adaptive immunity}

Ligation of TLRs by PAMPs leads to induction of cytokine production, and at first glance, the pathways involved are rather similar and seem to be inherently redundant. Hirschfeld and colleagues (2001) were the first to suggest that differential cytokine patterns are released when various TLRs are engaged by LPS from different species. This and additional studies showed that TLRs not only enable the innate immune system to recognize specific PAMPs, but by inducing specific cytokine profiles, bring a certain degree of specificity to the innate immune system and influence the nature of the adaptive immune responses. The capture of microbial antigens in the peripheral tissues and migration to the draining lymph nodes is the first step in the generation of adaptive immunity. Subsequent presentation of the antigen to naive T cells in the context of $\mathrm{MHC}$ molecules induces $\mathrm{T}$-cell activation and differentiation. It is now clear that both of these two steps in the initiation of adaptive immunity are under control of TLRs. In response to microbial pathogens, $\mathrm{CD} 4^{+}$Tcells differentiate into Th1 or Th2 cells. Each of these subsets is responsible for activating immune responses adapted to the type of infectious agent. Th1 cells produce IFN- $\gamma$ and induce $B$ cells to release antibodies of the immunoglobulin G2 isotype, responsible for phagocyte activation and antibody-dependent cellular cytotoxicity. Th2 cells produce IL-4, IL-5, and IL-10 and induce production of immunoglobulin $\mathrm{E}$ antibodies, responsible for immunity against parasitic infections (Jancovic et al., 2001). The uptake of microorganisms by DC through TLRs induces the up regulation of co-stimulatory and $\mathrm{MHC}$ molecules, a switch in the chemokine expression and migration to the draining lymph nodes (Banchereau and Steinman 1998).

The activation of $\mathrm{T}$ cells by antigen presenting cells such as DCs is the result of three distinct signals. Signal 1 derives from ligation of $\mathrm{T}$ cell receptors by specific pathogen peptides presented in the context of MHC class II molecules; signal 2 consists of the activation of co-stimulatory molecules (CD28 with CD80/86 and CD40 with CD154); and signal 3 is a polarizing signal given by specific cytokines, with IL-12 driving a Th1-type response, whereas IL-4 and IL-10 activate mainly Th2-type responses. The recognition and uptake of microorganisms through TLRs lead to the increased expression of the costimulatory molecules on the surface of DC membrane, which are the major signaling route for DC maturation (Medzhitov, 2001). Because presentation of an antigen in the absence of co-stimulatory signals leads to energy instead of activation of $\mathrm{T}$ cells, the stimulation of CD80 and CD86 by TLRmediated signals is a crucial step in the activation of adaptive immunity. Thus, TLRs participate in the translation of then on specific information contained in conserved PAMPs into antigen-specific information and clonal expansion of T cells.

Further tailoring of adaptive immunity occurs by the differentiation of activated $\mathrm{T}$ cells into either Th1 or Th2 cells. The most important signal responsible for T-helper cell differentiation is the type of cytokine profile present at the time of $\mathrm{T}$-cell stimulation.

The induction of this specific cytokine profile upon recognition of microbial pathogens greatly depends on recognition by specific TLRs. Thus, TLR agonists could be used as 
effective vaccine adjuvants to direct the immune response towards Th1 type which is a key consideration in development of modern vaccines.

\section{References}

Akira S. (2003) Mammalian Toll-like receptors. Current Opinions in Immunology 15, 5-11Aliprantis $\mathrm{O}$, Yang R, Weiss D, Godowski $\mathrm{P}$ and Zychlinsky A.(2000). Theapoptic signaling pathway activated by Toll like receptor-2. The European Molecular Biology Organization journal 19, 3325-3336.

Banchereau J and Steinman R. (1998) Dendritic cells and the control of immunity. Nature 392,245-252.

Barton G and Medzhitov R. (2002) Control of adaptive immune responses by Tolllike receptors. Current Opinion in Immunology14,380-383.

Chaudhary P, Ferguson C and Nguyen V. (1998) Cloning and characterization of two Toll/interleukin-1 receptor-like genes TIL3 and TIL4: evidence for a multi-gene receptor family in humans. Blood. 91, 4020-4027.

Gao B and Tsan M. (2003) Endotoxin contamination in recombinant human heat shock protein70 (Hsp70) preparation is responsible for the induction of tumor necrosis factor- $\alpha$ release by murine macrophages. Journal of Biological Chemistry. 278, 174.

Hirschfeld M, Weis J, Toshchakov V, Salkowski C, Cody M, Ward D, Qureshi N, Michalek M and Vogel N.(2001). Signaling by Toll-like receptor 2 and 4 agonists results in differential gene expression in murine macrophages. Infection and Immunity. 69, 1477.

Hopkins P, Fraser J and Pridmore A. (2005)
Super antigen recognition by HLA class II on monocytes up regulates tolllike receptor 4 and enhances proinflammatory responses to endotoxin. Blood 105, 3655-3662

Jancovic D, Liu Z and Gause W. (2001) Th1and Th2-cell commitment during infectious diseases:asymmetry in divergent pathways. Trends in Immunology22,450-457.

Janeway C and Medzhitov R. (2002) Innateimmune recognition. Annual Reviews of Immunology 20, 197-216.

Krug A, Luker G, Barchet W, Leib D, Akira S and Colonna M. (2004) Herpes simplex virus type 1 activates murine natural interferon-producing cells through toll-like receptor 9. Blood 103, 1433.

Lund J, Alexipoulou L, Sato A, Karow M, Adams N, Gale N, Iwasaki A and Flavell R. (2004) Recognition of single-stranded RNA viruses by Tolllike receptor 7. Proceedings of Natural Academy of Sciences U. S. A. 101, 5598-5603.

Medzhitov R. (2001) Toll like receptors and innate immunity. Natural Review of Immunology 1,135-145.

Menzies M and Ingham A. (2006) Identification and expression of Toll like receptors 1-10 in selected bovine and ovine tissues. Veterinary Immunology and Immunopathology 109, 23-30.

Netea M, van Deuren M, Kullberg, Cavaillon J and Van der Maer W. (2002) Does the shape of lipid A determine the interaction of LPS with Toll-like receptors? Trends in Immunology. 23, 135.

O’NeillL. (2002) Toll-like receptor signal transduction and the tailoring of innate immunity. Trends in Immunology23, 296-300.

Ooi J, Yagi Y, Hu X and Ip Y.(2002) The 
Drosophila Toll-9 activates a constitutive antimicrobial flybase. European Molecular Biology Organization Reports. 3,82-87.

Pichlmair A and Reis E.(2007). Innate recognition of viruses. Immunity 27: 370-383.

Schwandner R, Dziarski R, Wesche H, Rothe $M$ and Kirschning J. (1999). Peptidoglycan-and Lipoteichoic Acidinduced Cell Activation Is mediated by Toll-like receptor 2. The Journal of Biological Chemistry 274,1740617409.

Shah S M and Ravi K. (2014). Expression analysis of toll-like receptors 2 in bubaline peripheral blood mononuclear cells upon stimulation by B.Subtilis cell wall peptidoglycan. IOSR Journal of Agriculture and Veterinary Sciences 7, 39-43.

Shah S M, Kumar R, Brah G S, Santra L and Pawar H. (2012). Differentiation expression of Th1 and Th2-type cytokines in peripheral blood mononuclear cells of Murrah buffalo (Bubalus bubalis) on TLR2 induction by $B$. Subtilis peptidoglycan. AsianAust. J. Animal Sciences 25, 1021-
1028.

Shah S M, Kumar R and Brah G S. (2010). Identification and sequence analysis of TLR2 gene in Murrah buffalo. Online Journal of Veterinary Research 14, 218-226.

Smith K, Andersen N, Hayashi F, Strobe K, Bergman M, Barrett S, Cookson B and Aderem A. (2003)Toll-like receptor 5 recognizes a conserved site on flagellin required for protofilament formation and bacterial motility. Natural Immunology.4,1247.

Takeda K and Akira S. (2005) Toll-like receptors in innateimmunity. International Immunology 17, 1-14.

Vahanan B, Raj G, Pawar R, Gopinath V, Raja A and Thangavelu A. (2008) Expression profile of toll like receptors in a range of water buffalo tissues (Bubalus bubalis). Veterinary Immunology and Immunopathology 126,149-155.

Zhang D, Zhang G, Hayden M, Greenbalt M, Bussey C, Flavell $\mathrm{R}$ and Ghosh S. (2004) A Toll-like receptor that prevents infection again sturopathogenic bacteria. Science. 303,1522 .

\section{How to cite this article:}

Syed Mohmad Shah and Arem Qayum. 2021. Toll-Like Receptors-The Guardians at the Interface. Int.J.Curr.Microbiol.App.Sci. 10(07): 722-729. doi: https://doi.org/10.20546/ijcmas.2021.1007.078 